\title{
RELAÇÃO DAS COMORBIDADES EM INDIVÍDUOS OBESOS E A INFECÇÃO POR COVID-19
}

\section{Felipe Figueiredo Moreira1, ${ }^{1}$, Fernanda Emanuelle ViomarRocha2 ${ }^{2}$, Dayana Carolina Ribeiro ${ }^{3}$, Patricia Pacheco Tyski Suckow ${ }^{4}$, Angela Dubiela Julik ${ }^{5}$,Eliane Gonçalves de Jesus Fonseca ${ }^{6}$, Pamela Taina Licoviski ${ }^{7}$, Josiane Lopes ${ }^{8}$ and Ana Carolina Dorigoni Bini ${ }^{9}$}

1,2,3 Discente de Fisioterapia da Universidade Estadual do Centro-Oeste; 4,5,6,8,9 Docente do curso de Fisioterapia da Universidade Estadual do Centro-Oeste;

${ }^{7}$ Residente em Saúde do Idoso- HURCG/UEPG;

\section{ARTICLE INFO}

\section{Article History:}

Received $04^{\text {th }}$ March, 2021

Received in revised form

$02^{\text {nd }}$ April, 2021

Accepted $26^{\text {th }}$ May, 2021

Published online $30^{\text {th }}$ June, 2021

Key Words:

Obesidade, infecções por Coronavírus,

Comorbidade, Atividade Motora.

*Corresponding author:

Felipe Figueiredo Moreira

\begin{abstract}
Objetivo: Verificar por meio de uma revisão sistemática, a relação da obesidade e a nova doença causada pelo Covid-19. Métodos: Realizada uma pesquisa por meio de uma revisão sistemática, sobrea correlação do risco de contágio por Covid-19 em obesos, através das bases de dados Medline, PubMed, Scielo, Science Direct e Springer. Resultados: Foram selecionados 18 artigos que atendiam aos critérios, e que evidenciavam o alto risco gerado por comorbidades como a obesidade no contágio ao Covid-19. Conclusão: Podemos concluir que a obesidade é um fator de risco importante quando associado ao novo coronavírus (Covid-19), o que exige uma maior atenção às medidas preventivas em indivíduos suscetíveis. Enquanto não houver novas descobertas, devemos lembrar que fatores modificáveis do estilo de vida, como reeducação alimentar e atividade física devem ser priorizados.
\end{abstract}

Copyright (C) 2021, Felipe Figueiredo Moreira et al. This is an open access article distributed under the Creative Commons Attribution License, which permits unrestricted use, distribution, and reproduction in any medium, provided the original work is properly cited.

Citation: Felipe Figueiredo Moreira, Fernanda Emanuelle Viomar Rocha, Dayana Carolina Ribeiro et al. "Relação das comorbidades em indivíduos obesos e a infecção por covid-19”, International Journal of Development Research, 11, (06), 47993-47998.

\section{INTRODUCTION}

Denominado como Covid-19, a nova doença que se espalhou rapidamente de forma global, já conta com mais de 170 mil mortes e mais de 2 milhões de infectados no mundo pelo novo coronavírus SARS-Co-2, o qual foi inicialmente detectado na China (ZbindenFoncea et al., 2020). Responsável pelo Covid-19, o SARS-CoV-2 ${ }^{1}$, é um vírus que pertence à família Coronaviridae, composto por um ácido ribonucleico de fita simples (Sohrabi et al., 2020). Entre os sintomas mais recorrentes estão a febre, tosse seca, também dificuldade para respirar, há os casos onde pode haver sérias complicações, como edema pulmonar, choque séptico, falência de órgãos e síndrome do desconforto respiratório agudo (Chen, 2020), e também os casos onde não há o aparecimento de nenhum sintoma $(\mathrm{Wu}, 2020)$. Existem alguns fatores de risco que podem acabar aumentando a chance do indivíduo ficar mais susceptível ao novo vírus, alguns deles são pessoas com doenças cardiovasculares, hipertensão, diabetes, pessoas com média de 60 anos, tabagismo, doenças pulmonares e obesidade (Zbinden-Foncea et al., 2020). Com cerca de 650 milhões de pessoas obesas no mundo ${ }^{5}$, a obesidade se torna uma das doenças que mais se destaca dentre os riscos ao Covid19 (Kalligeros et al., 2020). Em um estudo realizado nos Estados Unidos com 5.700 pacientes com o novo coronavírus, cerca de $41 \%$ dentre eles eram obesos (Richarson et al., 2020), demonstrando uma alta porcentagem de pacientes com uma mesma comorbidade, o que gera um alerta a essa comunidade. De maneira fisiológica, os indivíduos obesos apresentam uma alta concentração de adipocina pró-inflamatória, e baixa concentração de adipocina anti-inflamatória, gerando um ambiente de risco pela também baixa produção de TCD8 , que é responsável por grande parte na proteção contra novos vírus (Luzi, 2020). Assim, a obesidade acaba desenvolvendo maior vulnerabilidade a infecções, logo, também ao vírus SARS-CoV-2 (Misumi, 2019), sendo associada as formas mais graves da doença do Covid-19. Por ser de origem inflamatória a obesidade, leva a uma resposta baixa da imunidade nesses indivíduos (Malavazos, 2020), a qual se mostra altamente prejudicada, pois existe certa alteração no desenvolvimento de células como linfócitos, na resposta imune, tanto inata como na adaptativa, e um maior risco de processos inflamatórios 
criado pelo aumento de adipócitos (Phillips, 2020), fazendo com que o indivíduo nessas condições também não tenha uma melhor resposta a vacinas ou antivirais (Muscogiuri, 2020). Em contrapartida a essa baixa resposta da imunidade pela obesidade, tem-se pesquisado muito sobre intervenções que melhorem a defesa imune do nosso corpo, entre eles uma dieta nutricional adequada e exercícios tem-se mostrado como grandes aliados para aumentar positivamente essa defesa $^{13}$. Os exercícios por exemplo são ótimos no recrutamento de células T, células Natural Killer (Niemam, 2018), aumento de IL-6 e IL-10 gerando efeitos anti-inflamatórios (Pedersen, 2017), que combinados a uma boa nutrição podem trazer grandes benefícios para o sistema imunológico (Rahmati-Ahmadabad, 2020). Essa revisão teve como objetivo verificar os artigos que demonstram as principais relações entre o Covid-19 e a obesidade. Compreendendo o porquê de pacientes obesos estarem em evidência nas zonas de riscos para o SARS-CoV-2, assim como possíveis medidas que colaboram para o fortalecimento da barreira imune do nosso corpo.

\section{METODOLOGIA}

Trata-se de revisão sistemática elaborada por meio do protocolo Prisma, através das bases de dados Medline, PubMed, Scielo, Science Direct e Springer, de forma totalmente independente. A identificação do tema baseou-se na correlação da obesidade com COVID-19, para isso foram usados os descritores: Obesidade, Covid-19, Atividade Física e seus respectivos em inglês. Houve a identificação dos estudos pré-selecionados e selecionados, seguindo os critérios de inclusão, sendo excluídos aqueles em que a obesidade não tinha relação alguma com o Covid-19. O próximo passo a partir dos artigos selecionados teve suas metodologias, resultados, discussões e conclusões analisados e discutidos, apresentada a revisão/síntese do conteúdo abordado. Nos respectivos artigos analisados nenhum procedimento de risco foi feito em indivíduos. Foram encontrados 30 artigos. De acordo com os seguintes critérios de inclusão, 18 artigos foram selecionados. Com a finalidade de uma maior compreensão da metodologia escolhida, a seguir foram apresentadas de modo sistemático cada etapa da Revisão, utilizada para a elaboração deste estudo (Figura 1).

Qualidade da Evidência: A avaliação da qualidade da evidência pelo Sistema Grading of Recommendations Assessment, Developmentand Evaluation (GRADE), proporcionando maior confiabilidade à análise crítica dos achados. Conforme o sistema GRADE, os estudos foram classificados em quatro categorias de qualidade da evidência:

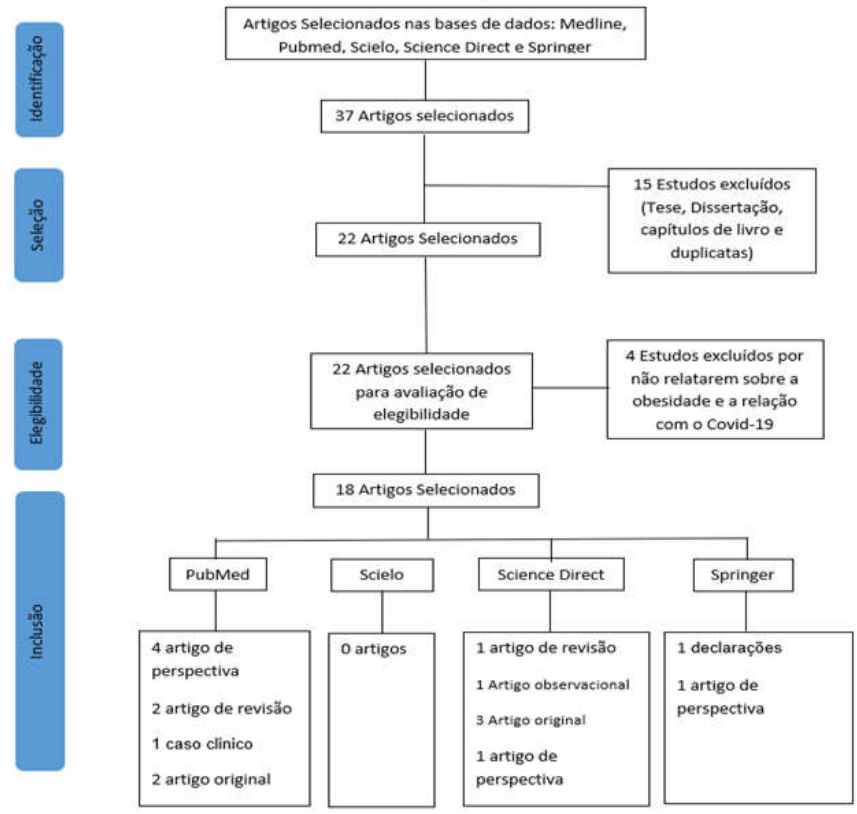

Alta - Há forte confiança de que o verdadeiro efeito/associação esteja próximo do estimado; Moderada - Há confiança moderada no efeito/associação estimado; Baixa - A confiança no efeito/associação é limitada; Muito Baixa - A confiança na estimativa do efeito/associação é muito limitada.

\section{RESULTADOS}

Os artigos foram estudados e analisados criteriosamente, sendo apenas utilizados aqueles com tratamento em humanos ou com recomendações ou perspectiva ou declaração, selecionando somente 18 que atenderam aos critérios dessa revisão (Tabela 1).

\section{DISCUSSÃO}

Tem-se observado que pessoas com excesso de o peso corporal podem apresentar uma infecção mais grave por COVID-19 (Rychter, 2020). De acordo com o trabalho realizado por Stefan ${ }^{16}$ existe uma grande preocupação relacionada com o IMC e pacientes com diagnóstico de Covid-19. O IMC $>40 \mathrm{~kg} / \mathrm{m}^{2}$ foi o segundo preditor independente mais forte de hospitalização ${ }^{16}$. Adultos com obesidade mais grave (definida por maior IMC) e aqueles com obesidade central (definida por maior circunferência da cintura ou maior proporção cintura-quadril) estão em um risco maior de desenvolver COVID-19 grave (Zhao, 2020). Os parâmetros que medem esse alto risco é a relação da obesidade associada com uma mecânica respiratória prejudicada, aumento da resistência das vias aéreas, além de outras características fisiopatológicas da obesidade como a força muscular respiratória e volumes pulmonares ${ }^{16}$, assim como a associação com a alveolite destrutiva, disfunção do surfactante, síndrome da apneia do sono e deterioração do controle glicêmico, o que pode comprometer a função ventilatória e contribuir para um cenário de piora no caso da infecção por Covid-19 (Puig-Domingo, 2020). Zbinden-Foncea apresentou evidências preliminares despontando que a gravidade dos sintomas associados ao Covid-19 e o resultado final da infecção por esse vírus estão associados ao estado de saúde dos indivíduos antes da infecção ${ }^{1}$. Sedentarismo ou atividade física reduzida são características de pacientes obesos em relação a indivíduos magros ${ }^{8}$. Doenças relacionadas ao estilo de vida, sustentadas por padrões de comportamento sedentário, maus hábitos alimentares e falta de exercício físico, são fatores ligados ao alto risco de hospitalização e mortalidade de pacientes que estão sobrepeso ou obesos. Embora o Covid-19 seja notoriamente contagioso, também parece ser preferencialmente virulento entre adultos mais velhos ( $>60$ anos) com comorbidades existentes ${ }^{18}$, em um estudo realizado por Hernández-Garduño foi utilizado o banco de dados Covid-19 disponível publicamente do Ministério da Saúde mexicano por meio do site "Dirección General de Epidemiología", do qual ele obteve informações de todos os pacientes avaliados para Covid-19 em 15 de maio de 2020, seu achados indicaram que a obesidade é a comorbidade preditora mais forte para o Covid-19 entre os mexicanos, seguida por diabetes e hipertensão (Hernández-Garduño, 2020). Provavelmente, muitos desses fatores estão intimamente ligados à resistência à insulina e gordura visceral (Halpern, 2020). Durante a pandemia do vírus influenza A (H1N1) em 2009, a obesidade também foi associada ao aumento do risco de doença grave e mortalidade. Os mecanismos que ligam o mau prognóstico de COVID-19 à obesidade se sobrepõem às vias que regulam a função imunológica (Lim, 2020), incluindo a interrupção da integridade do tecido linfóide, alterações no desenvolvimento de leucócitos, atividade dos fenótipos, e a coordenação de respostas imunes inatas e adaptativas, apresentando também problemas mecânicos relacionados ao excesso de peso que dificultam o diagnóstico precoce com ultrassonografia pulmonar. Em um estudo realizado por Richardson ${ }^{7} \mathrm{e}$ seus colaboradores, foram incluídos 5700 pacientes com diagnóstico positivo pra Covid-19, onde as comorbidades mais comuns encontradas foram hipertensão $(56,6 \%)$, obesidade $(41,7 \%)$ e diabetes $(33,8 \%)$. Em outro estudo realizado por Simmonet ${ }^{19}$ e colaboradores, a obesidade (IMC $>30 \mathrm{~kg} / \mathrm{m} 2$ ) e a obesidade grave (IMC> $35 \mathrm{~kg}$ / $\mathrm{m} 2$ ) também foram significativamente frequentes entre os 124 pacientes admitidos com Covid- $19^{19}$. 


\begin{tabular}{|c|c|c|c|c|c|c|c|}
\hline \multicolumn{7}{|c|}{ Avaliação da Qualidade } & \multirow[t]{2}{*}{ Qualidade (GRADE) } \\
\hline Estudos & Delineamento & Limitações metodológicas & Inconsistência & Evidencia indireta & Imprecisão & $\begin{array}{l}\text { Outras } \\
\text { Considerações }\end{array}$ & \\
\hline Stefan, et al. 2020 & Artigo de perspectiva & limitações graves & Sem Inconsistência grave & Sem Evidencias indiretas importantes & Imprecisão grave & Nenhuma & BAIXO \\
\hline Puig-Domingo, et al. 2020 & Declaração & limitações graves & Sem Inconsistência grave & Sem Evidencias indiretas importantes & Imprecisão grave & Nenhuma & BAIXO \\
\hline Zbinden-Foncea, et al. 2020 & Artigo de revisão & Sem limitações graves & Sem Inconsistência grave & Sem Evidencias indiretas importantes & Imprecisão grave & Nenhuma & MODERADO \\
\hline Luzi, et al. 2020 & Artigo de perspectiva & limitações graves & Sem Inconsistência grave & Sem Evidencias indiretas importantes & Imprecisão grave & Nenhuma & BAIXO \\
\hline Carter, et al 2020 & Artigo de perspectiva & limitações graves & Sem Inconsistência grave & Sem Evidencias indiretas importantes & Imprecisão grave & Nenhuma & BAIXO \\
\hline Muscogiuri, et al 2020 & Artigo observacional & limitações graves & Sem Inconsistência grave & Sem Evidencias indiretas importantes & Imprecisão grave & Nenhuma & BAIXO \\
\hline Richarson, et al. 2020 & Artigo de caso clinico. & Sem limitações graves & Sem Inconsistência grave & Sem Evidencias indiretas importantes & Sem Imprecisão grave & Nenhuma & ALTO \\
\hline Simonnet, et al 2020 & Artigo original. & limitações graves & Sem Inconsistência grave & Sem Evidencias indiretas importantes & Sem Imprecisão grave & Nenhuma & MODERADO \\
\hline Malavazos, et al 2020 & Artigo de perspectiva. & limitações graves & Sem Inconsistência grave & Sem Evidencias indiretas importantes & Imprecisão grave & Nenhuma & BAIXO \\
\hline Zeng, et al 2020 & Artigo original. & Sem limitações graves & Sem Inconsistência grave & Sem Evidencias indiretas importantes & Imprecisão grave & Nenhuma & MODERADO \\
\hline $\begin{array}{l}\text { Rahmati-Ahmadabad, et al. } \\
2020\end{array}$ & Artigo de revisão & Sem limitações graves & Sem Inconsistência grave & Sem Evidencias indiretas importantes & Imprecisão grave & Nenhuma & MODERADO \\
\hline Zhao, et al. 2020 & Artigo original. & Sem limitações graves & Sem Inconsistência grave & Sem Evidencias indiretas importantes & Sem Imprecisão grave & Nenhuma & ALTO \\
\hline Kimura, et al. 2020 & Artigo de perspectiva. & limitações graves & Sem Inconsistência grave & Sem Evidencias indiretas importantes & Imprecisão grave & Nenhuma & BAIXO \\
\hline $\begin{array}{l}\text { Hernández-Garduño, et al. } \\
2020\end{array}$ & Artigo original. & Sem limitações graves & Sem Inconsistência grave & Sem Evidencias indiretas importantes & Sem Imprecisão grave & Nenhuma & ALTO \\
\hline Rychter., et al. 2020 & Revisão sistemática. & Sem limitações graves & Sem Inconsistência grave & Sem Evidencias indiretas importantes & Sem Imprecisão grave & Nenhuma & ALTO \\
\hline Halpern, et al. 2020 & Artigo de perspectiva & limitações graves & Sem Inconsistência grave & Sem Evidencias indiretas importantes & Imprecisão grave & Nenhuma & BAIXO \\
\hline Holly, et al. 2020 & Artigo original. & limitações graves & Sem Inconsistência grave & Sem Evidencias indiretas importantes & Sem Imprecisão grave & Nenhuma & MODERADO \\
\hline Lim, et al. 2020 & Relatório. & limitações graves & Sem Inconsistência grave & Sem Evidencias indiretas importantes & Imprecisão grave & Nenhuma & BAIXO \\
\hline
\end{tabular}

Tabela 1. Descrição dos artigos originais da discussão

\begin{tabular}{|c|c|c|c|}
\hline Autor (es) / ANO & Amostra & Resultados & Conclusões \\
\hline Stefan, et al. 2020 & Artigo de perspectiva & $\begin{array}{l}\text { Embora seja amplamente reconhecido que a presença de comorbidades como } \\
\text { hipertensão, diabetes mellitus e DCV estão associados a um curso mais grave do } \\
\text { COVID-19, a obesidade dificilmente foi investigada. }\end{array}$ & $\begin{array}{l}\text { A obesidade é o principal fator de risco para essas comorbidades, } \\
\text { geralmente por problemas de saúde metabólica. Essas medições são cruciais } \\
\text { para estimar melhor o risco de complicações em pacientes com COVID-19. }\end{array}$ \\
\hline $\begin{array}{l}\text { Puig-Domingo, et al. } \\
2020\end{array}$ & $\begin{array}{l}\text { Uma declaração do Parlamento Europeu } \\
\text { Sociedade de Endocrinologia }\end{array}$ & $\begin{array}{l}\text { Jovens nos quais a obesidade grave é presente pode evoluir para alveolite destrutiva } \\
\text { com insuficiência respiratória. Além disso a obesidade grave é associada à síndrome da } \\
\text { apneia do sono, bem como a disfunção sulfactante que pode contribuir para um caso de } \\
\text { infecção por COVID-19. }\end{array}$ & $\begin{array}{l}\text { Esses pacientes podem estar em maior risco de resultados prejudicados no } \\
\text { caso de infecção por COVID-19. }\end{array}$ \\
\hline $\begin{array}{l}\text { Zbinden-Foncea, et al. } \\
2020\end{array}$ & Artigo de revisão & $\begin{array}{l}\text { Indivíduos com obesidade, geralmente apresentam inflamação crônica caracterizada } \\
\text { por aumento níveis de várias citocinas pró-inflamatórias: esse estado predispõe a maior } \\
\text { risco de infecção, juntamente com mais resultados adversos. }\end{array}$ & $\begin{array}{l}\text { Entre os fatores ligados ao aumento do risco de hospitalização são } \\
\text { obesidade, resistência à insulina e diabetes: essas doenças, sustentada por } \\
\text { padrões de comportamento sedentário são caracterizados por inflamação } \\
\text { crônica O que pode ser prejudicial em um caso de infecção por COVID-19. }\end{array}$ \\
\hline Luzi, et al. 2020 & Artigo de perspectiva & $\begin{array}{l}\text { Grande prevalência de indivíduos obesos na população pode aumentar a chance de } \\
\text { surgir uma cepa viral mais virulenta, prolonga o derramamento de vírus por todo a } \\
\text { população e, eventualmente, pode aumentar a taxa geral de mortalidade da pandemia } \\
\text { do COVID-19. }\end{array}$ & $\begin{array}{l}\text { A quarentena em indivíduos obesos provavelmente deve ser maior do que } \\
\text { em indivíduos com peso normal. }\end{array}$ \\
\hline Carter, et al 2020 & Artigo de perspectiva & $\begin{array}{l}\text { A forma biologicamente ativa da vitamina } \mathrm{D} \text { tem sido implicada em várias doenças } \\
\text { inflamatórias, infecciosas e pulmonares. A suplementação diária ou semanal de } \\
\text { vitamina } \mathrm{D} \text { também demonstrou oferecer proteçãa contra infecções respiratórias aguda. }\end{array}$ & $\begin{array}{l}\text { A vitamina D pode ser um complemento para o meio pró-inflamatório } \\
\text { observada em pacientes com COVID-19. Pois as implicações clínicas dos } \\
\text { sintomas severidade parecem exacerbados em indivíduos com hipertensão e } \\
\text { diabetes, ambos estão ligados à obesidade. }\end{array}$ \\
\hline Muscogiuri, et al 2020 & Artigo observacional & $\begin{array}{l}\text { Resultados recentes destacaram o impacto que a obesidade tem sobre a imunidade e } \\
\text { defesa de patógenos, interrupção de integridade do tecido linfoide, alterações no } \\
\text { desenvolvimento de leucócitos, coordenação da resposta imune inata e adaptativa. }\end{array}$ & $\begin{array}{l}\text { A obesidade também demonstrou prejudicar a memória das respostas das } \\
\text { células TCD8+ à infecção pelo vírus influenza. }\end{array}$ \\
\hline Richarson, et al. 2020 & $\begin{array}{l}\text { Artigo de caso clinico, incluídos } 5700 \\
\text { pacientes com COVID-19 }\end{array}$ & Foram incluídos 5700 pacientes com COVID-19, destes, 41,7\% tinhaobesidade (1737). & $\begin{array}{l}\text { A obesidade esta entre as posições mais altas das comorbidades com fator de } \\
\text { risco ao COVID- } 19 \text {. }\end{array}$ \\
\hline
\end{tabular}




\begin{tabular}{|c|c|c|c|}
\hline Simonnet, et al 2020 & $\begin{array}{l}\text { Artigo original, analisando a relação } \\
\text { entre fatores clínicos, características } \\
\text { físicas, incluindo indice de massa } \\
\text { corporal (IMC), e a exigência de invasão } \\
\text { mecânica (IMV) em 124 pacientes } \\
\text { consecutivos admitidos em terapia } \\
\text { intensiva por SARS-CoV-2 }\end{array}$ & $\begin{array}{l}\text { Obesidade (IMC> } 30 \mathrm{~kg} / \mathrm{m} 2) \text { e obesidade grave (IMC> } 35 \mathrm{~kg} / \mathrm{m} 2) \text { estiveram } \\
\text { presentes em } 47,6 \% \text { e } 28,2 \% \text { dos casos, respectivamente. A proporção de pacientes que } \\
\text { necessitaram de IMV foi maior em pacientes com IMC }>35 \mathrm{~kg} / \mathrm{m} 2(85,7 \%)\end{array}$ & $\begin{array}{l}\text { O estudo mostrou alta frequência de obesidade entre os pacientes admitidos } \\
\text { em cuidados intensivos para SARS-CoV-2. }\end{array}$ \\
\hline Malavazos, et al 2020 & Artigo de perspectiva & $\begin{array}{l}\text { As pessoas com obesidade desenvolverem mais complicações se expostas a um vírus } \\
\text { podem ser atribuídas ao estado crônico inflamatório e resposta imunológica retardada e } \\
\text { ineficaz. Múltiplos mecanismos o tecido adiposo pode contribuir para o } \\
\text { desenvolvimento e progressão da doença de COVID-19 }\end{array}$ & $\begin{array}{l}\text { O papel do tecido adiposo durante doenças infecciosas, pode ser } \\
\text { importante.Ações terapêuticas direcionadas ao tecido adiposo podem ser } \\
\text { consideradas para reduzir a carga da doença COVID-19. }\end{array}$ \\
\hline Zeng, et al 2020 & $\begin{array}{l}\text { Artigo original, } 214 \text { pacientes com } \\
\text { COVID-19 confirmado em laboratório } \\
\text { com idade entre } 18 \text { e } 75 \text { anos de três } \\
\text { hospitais em Wenzhou, China. }\end{array}$ & $\begin{array}{l}\text { A presença de obesidade em pacientes com DMEM (doença hepática gordurosa } \\
\text { associada ao metabolismo) foi associada a aproximadamente } 6 \text { vezes aumento do risco } \\
\text { de doença COVID-19 grave. }\end{array}$ & $\begin{array}{l}\text { Os dados demonstram que o risco de obesidade para a gravidade do COVID- } \\
19 \text { é maior naqueles com DMEM. }\end{array}$ \\
\hline $\begin{array}{l}\text { Rahmati-Ahmadabad, } \\
\text { et al. } 2020\end{array}$ & Artigo de revisão & A obesidade é um dos maiores fatores de risco relacionados ao COVID-19. & $\begin{array}{l}\text { Exercícios de intensidade moderada devem ser recomendado como um } \\
\text { medicamento não farmacológico, barato, e de maneira viável de lidar com o } \\
\text { vírus COVID-19. }\end{array}$ \\
\hline Zhao, et al. 2020 & $\begin{array}{l}\text { Artigo original, examinar as associações } \\
\text { de características relacionadas à } \\
\text { obesidade e sua predisposição genética } \\
\text { com o risco de desenvolver COVID-19 } \\
\text { grave em } 489.769 \text { adultos matriculados } \\
\text { no UK Biobank. }\end{array}$ & $\begin{array}{l}\text { Foi identificado } 641 \text { pacientes com COVID-19 grave. Comparado com adultos com } \\
\text { peso normal, aqueles com um IMC mais alto tiveram um aumento de dose-resposta no } \\
\text { risco de COVID-19 grave. }\end{array}$ & $\begin{array}{l}\text { A predisposição genética para a obesidade apresenta maior risco de } \\
\text { desenvolver COVID-19 grave. }\end{array}$ \\
\hline Kimura, et al. 2020 & Artigo de perspectiva & $\begin{array}{l}\text { Aumento do tecido adiposo intra-abdominal comprime fisicamente os pulmões. A } \\
\text { redução da complacência pulmonar e a contração dos pulmões resulta no encurtamento } \\
\text { do diâmetro interno das vias aéreas e das vias aéreas músculo liso. Depósitos de } \\
\text { gordura nos tecidos moles da faringe aumenta a resistência inspiratória, resultando em } \\
\text { diminuição da pressão das vias aéreas. A obesidade aumenta o risco de complicações } \\
\text { da síndrome da apnéia do sono. }\end{array}$ & $\begin{array}{l}\text { Uma melhor compreensão dos mecanismos pelos quais a obesidade contribui } \\
\text { para a gravidade de COVID-19 é importante para o desenvolvimento de } \\
\text { tratamentos mais eficazes. }\end{array}$ \\
\hline $\begin{array}{l}\text { Hernández-Garduño, et } \\
\text { al. } 2020\end{array}$ & $\begin{array}{l}\text { Artigo original, estudo usou o banco de } \\
\text { dados Covid-19 disponível publicamente } \\
\text { no Ministério da Saúde do México. Tipo } \\
\text { de paciente: ambulatorial ou } \\
\text { hospitalizado ou internado em unidade } \\
\text { de terapia intensiva (UTI) ou tinha sido } \\
\text { intubado. }\end{array}$ & Um total de 32.583 pacientes hospitalizados foram identificado com uma comorbidade. & Obesidade representou o preditor mais forte para Covid-19. \\
\hline Rychter., et al. 2020 & Revisão sistemática. & $\begin{array}{l}\text { A obesidade é cada vez mais considerada como mais um fator de risco, porque foi } \\
\text { observado que pessoas que sofrem de corpo excessivo de peso podem apresentar um } \\
\text { curso mais grave de infeç̧ão por COVID-19. }\end{array}$ & $\begin{array}{l}\text { Perda de peso, deve ser considerada como uma dasmetas para prevenção de } \\
\text { cuidados e estratégia viável. Deve-se adotar como padrão realizar a medição } \\
\text { do peso corporal(com a avaliação do tecido adiposo) em cada paciente } \\
\text { COVID-19. }\end{array}$ \\
\hline Halpern, et al. 2020 & Artigo de perspectiva & $\begin{array}{l}\text { Evidências apontam que a perda de peso acima de } 10 \% \text { pode reduzir significativamente } \\
\text { riscos para a saúde, independentemente do IMC final. }\end{array}$ & $\begin{array}{l}\text { Menores perdas de peso e aumento da atividade física podem reduzir os } \\
\text { riscos de infecção por COVID- } 19 \text {. }\end{array}$ \\
\hline Holly, et al. 2020 & $\begin{array}{l}\text { Artigo original. Estudo de análise global } \\
\text { do COVID-19 na obesidade. }\end{array}$ & $\begin{array}{l}\text { Existem muitas características da obesidade que podem acentuar a resposta clínica à } \\
\text { infecção por SARS-CoV-2: comprometimento da resposta imune, estado } \\
\text { aterotrombótico, acúmulo de fim de glicação avançada e um estado inflamatório } \\
\text { crônico. }\end{array}$ & $\begin{array}{l}\text { Na obesidade mecanismos são ativados cronicamente devido à deterioração } \\
\text { metabolic estado e isso pode fornecer uma oportunidade maior para um mais } \\
\text { profundo e sustentado infecção viral. }\end{array}$ \\
\hline Lim, et al. 2020 & Relatório. & $\begin{array}{l}\text { Pessoas com obesidade deveriammanter um estilo de vida saudável, exercício regular e } \\
\text { uma alimentaçãosaudável são cruciais para fortalecer osistema imunológico e redução } \\
\text { da inflamação. }\end{array}$ & $\begin{array}{l}\text { Obesidade é um fator de risco para a infecção viral devido aos efeitos } \\
\text { deletérios da obesidade sobre o sistema imunológico. }\end{array}$ \\
\hline
\end{tabular}


O que corrobora com o estudo de coorte retrospectivo realizado por Muscogiuri ${ }^{11}$ e colaboradores em um único centro francês, também foram avaliados 124 pacientes, internados em terapia intensiva por SARS-CoV-2, foi observado que obesidade (IMC> $30 \mathrm{~kg} / \mathrm{m} 2$ ) e obesidade grave (IMC $>35 \mathrm{~kg} / \mathrm{m} 2$ ) estiveram presentes em $47,6 \%$ e $28,2 \%$ dos casos, respectivamente ${ }^{11}$. De acordo com as perspectivas de Malavazos ${ }^{10}$ a OMS considera as doenças não transmissíveis (DNTs), como a obesidade, um fator de risco importante para adoecer gravemente com o novo coronavírus de 2019. Um estudo do Centro Nacional de Pesquisa e Auditoria de Cuidados Intensivos do Reino Unido indicou que dois terços das pessoas que desenvolveram complicações graves ou fatais relacionadas ao Covid-19 estavam com sobrepeso ou obesidade $^{10}$. No estudo realizado por Zheng (Zheng, 2020) foram inscritos consecutivamente 214 pacientes positivos para Covid-19, com idades entre 18 e 75 anos, de três hospitais em Wenzhou, China para analisar a relação entre o Covid-19 e doença hepática gordurosa associada ao metabolismo. A associação entre obesidade (como exposição) e gravidade do Covid-19 (como resultado) entre pacientes com doença hepática gordurosa associada ao metabolismo foi avaliada por regressão logística binária. Os pacientes obesos apresentaram níveis mais altos de aspartato aminotransferase, glicemia de jejum e LDL-colesterol e menor número de linfócitos. Notavelmente, os pacientes com doença hepática gordurosa associada ao metabolismo que eram obesos tinham doença de Covid-19 mais grave ${ }^{20}$, o que corrobora com o estudo realizado por Holly, onde ele afirma que distúrbios metabólicos podem promover uma resposta clínica mais grave ao SARS-CoV-2 (Holly, 2020). Se a obesidade representa um preditor de mau prognóstico ou complicações de taxa mais alta em pacientes com SARS-Cov2, ainda é um fator de risco modificável (Malavazos, 2020). Intervenções de atividade física demonstraram o potencial de reduzir o risco de complicações ${ }^{8}$, a alta aptidão cardiorrespiratória e o treinamento aeróbico de intensidade moderada melhoram as respostas imunológicas à vacinação, diminuem a inflamação crônica de baixo grau e melhoram vários processos de marcadores imunológicos. Sugere-se que a função imune seja aumentada pela atividade física apropriada antes da doença, o que impulsionará o organismo contra o vírusafirma o artigo publicado por Rahmati-Ahmadabad (Rahmati-Ahmadabad, 2020).

\section{CONCLUSÃO}

Com base nos resultados obtidos através desta pesquisa bibliográfica, podemos concluir que a obesidade é um fator de risco importante quando associado ao novo coronavírus (Covid19), o que exige uma maior atenção às medidas preventivas em indivíduos suscetíveis. Enquanto não houver novas descobertas, devemos lembrar que fatores modificáveis do estilo de vida, como reeducação alimentar e atividade física devem ser priorizados.

\section{REFERÊNCIAS}

Carter SJ, Baranauskas MN, Fly AD. Considerations for obesity, vitamin D, and physical activity amidst the COVID-19 pandemic. Obesity (Silver Spring). 2020 Apr16;:1-6.

Chen N, Zhou M, Dong X, Qu J, Gong F, Han Y, et al. Epidemiological and clinical characteristics of 99 cases of 2019 novel coronavirus pneumonia in Wuhan, China: a descriptive study. Lancet. 29 Jan 2020. 1-7.

Halpern, B., Mancini, M.C. Controlled obesity status: a rarely used concept, but with particular importance in the COVID-19 pandemicandbeyond. J EndocrinolInvest 2020; 44(4), 877880.
Hernández-Garduño, E. Obesity is the comorbidity more strongly associated for Covid-19 in Mexico. A case-control study, Obesity Research \& Clinical Practice, 2020; 14: 375-379.

Holly JMP, Biernacka K, Maskell N andPerks CM (2020) Obesity, Diabetes and COVID-19: An Infectious Disease Spreading From the East Collides With theConsequences of an Unhealthy Western Lifestyle. Front. Endocrinol., 2020; 11:582870.

Honce R, Schultz-Cherry S. Impacto f Obesity on Influenza A Virus Pathogenesis, Immune Response, and Evolution. Front Immunol. 10 May 2019. 1-15.

Kalligeros M, Shehadeh F, Mylona KE, Benitez G, Beckwith GC, Chan PA. et al. Association of Obesity with Disease Severity among Patients with COVID-19. Obesity (Silver Spring). 2020 Apr 30. 1-13.

Kimura T, Namkoong H, Susceptibility of the obese population to COVID-19, International Journal of Infectious Diseases, 2020; 101:380-381.

Lim S, Shin SM, Nam GE, Jung CH, Koo BK. Proper Management of People with Obesity duringthe COVID-19 Pandemic. JOMES 2020;29:84-98.

Luzi L, RadaelliGM. Influenza and obesity: its odd relationship and the lessons for COVID-19 pandemic. Acta Diabetol. 2020 Apr 05;:1-6.

Malavazos AE, Romanelli MMC, Bandera F, Iacobellis G. Targeting the Adipose Tissue in COVID-19. Obesity (Silver Spring). 2020 Apr $21 ;: 1-7$.

Misumi I, Starmer J, Uchimura T, Beck MA, Magnuson T, Whitmire JK Obesity Expands a Distinct Population of $\mathrm{T}$ Cells in Adipose Tissue and Increases Vulnerability to Infection. Cell Rep. 9 Apr 2019. 1-17.

Muscogiuri G, Pugliese G, Barrea L, Savastano S, Colao A. Obesity: The "Achilles heel" for COVID-19?.Metabolism. 2020 Apr 25;108:1-4.

Niemam CD, Wentz LM. The compelling link between physical activity and the body's defense system. J Sport HearlthSci. 2018 Sep. 1-50.

Pedersen BK. Anti-inflammatory effects of exercise: role in diabetes and cardiovascular disease. Eur $\mathrm{J}$ of Clin Invest. 2017. 1-12.

Phillips CL, Grayson BE. The imune remodel: Weight lossmediated inflammatory changes to obesity. Exp Biol Med (Maywood). 2020. 1-13.

Puig-Domingo M, Marazuela M, Giustina A. COVID-19 and endocrine diseases. A statement from the European Society of Endocrinology. Endocrine. 2020 Abr; 68:2-5.

Rahmati-Ahmadabad S, Hosseini F. Exercise against SARS-CoV2 (COVID-19): Does workout intensity matter? (A mini review of some indirect evidence related to obesity). Obes Med. 2020 Apr24;:1-14.

Richarson S, Hirsch SJ, Narasimhan M, Crawford MJ, Mcginn T, Davidson WK, et al. Presenting Characteristics, Comorbidities, and Outcomes Among 5700 Patients Hospitalized With COVID-19 in the New York City Area. Jama. American Medical Association (AMA). 22 Apr 2020. 18.

Rychter AM, Zawada A,Ratajczak AE, Dobrowolska A, KrelaKaźmierczak I. Should patients with obesity be more afraid of COVID-19?.Obesity Reviews. 2020;1-8.

Simonnet A, Mikael Chetboun M, Poissy J, et al. High prevalence of obesity in severe acute respiratory syndrome coronavirus-2 (SARS-CoV- 2) requiring invasive mechanical ventilation. Obesity (silver spring). 2020 Apr 09;:1-15.

Sohrabi C, Alsafib Z, O’Neill N, Khan M, Kerwan A, Al-Jabir A. et al. World health Organization declares Global Emergency: A review of the 2019 Novel Coronavirus (COVID-19). Int J Surg. 2020 Fev. 1-19. 
Stefan N, Birkenfeld AL, Schulze MB., Ludwig DS. Obesity and impaired metabolic health in patients with COVID-19. Nat RevEndocrinol. 2020.

Wu Z, McGoogan JM. Characteristics of and Important Lessons From the Coronavirus Disease 2019 (COVID-19) Outbreak in China: Summary of a Reporto f 72314 Cases From the Chinese Center for Disease Control and Prevention. JAMA. 2020. 1-4.

Zbinden-Foncea H, Francaux M, Deldicque L, Hawley JA. Does high cardiorespiratory fitness confer some protection against pro-inflammatory responses after infection by SARS-CoV2?.Obesity (Silver Spring). 2020 Apr23;:1-14.
Zhao ZZ, Kohei Hasegawa, Baoshan Ma, MichimasaFujiogi, Carlos A. Camargo, Liming Liang. Association of obesity and its genetic predisposition with the risk of severe COVID-19: Analysis of population-based cohort data, Metabolism, 2020;112:154345.

Zheng KI, Gao F, Wang XB, et al. Obesity as a risk factor for greater severity of COVID-19 in patients with metabolic associated fatty liver disease. Metabolism. 2020 Apr 17;:1-16. 